\title{
AN EXPERIMENTAL STUDY OF TENDON GROWTH IN THE RABBIT
}

\author{
G. N. C. Crawford, Oxford, Exglaid \\ From the Department of Human Anatomy, Eniversity of Oxford
}

The post-natal growth of muscles and tendons is of interest both from the anatomical and from the orthopaedic point of view. But whereas much information is available on the growth and especially the differentiation of muscle, tendinous tissue has been relatively neglected. In fact, current opinion about tendon growth is largely based on the analogy of muscle.

Present knowledge of the development of muscle and tendon has resulted mainly from histological examination of material from young animals. Many authors have described the appearance of the end of a growing muscle belly where it joins the tendon, and the literature has been reviewed by Prenant, Bouin and Maillard (1911) and by Häggquist (1931). In the undifferentiated end of a muscle fibre there is a high concentration of nuclei and this is regarded as the region of most rapid muscle growth. Felix (1889) nevertheless believed that in the foetus some growth occurs throughout the length of the muscle fibre. The high concentration of nuclei extends into the tendon near its junction with the muscle, and it has been assumed that this indicates a multiplication of fibroblast nuclei which must be accompanied by a rapid deposition of collagen fibres. It seems unlikely, however, that the end of the tendon is the only site of fibroblast proliferation; and it would be reasonable to expect that new collagenous tissue might be laid down throughout the whole length of the tendon, and a means of interstitial longitudinal growth thus provided. Nevertheless this is not a generally accepted opinion (Haines 1932). Mason and Shearon (1932), investigating the capacity of tendinous tissue to form new collagenous fibres, divided the tendons of adult rabbits and found that although the greater part of the repair was accomplished by fibroblasts invading the lesion from without, some new collagen was laid down and organised into normal tendinous tissue by intrinsic fibroblasts. They thus showed that even in the adult new tendon fibres can originate from cells lying along the length of the tendon.

In dealing with a relatively slow dynamic process such as tendon growth, the examination of histological sections may be misleading. Additional sources of information should be sought, and a second possible approach to the problem is to examine living muscles and tendons in situ in the growing animal. Speidel (1938) observed the myomeres in the tails of living tadpoles over a long period and confirmed the hypothesis that the main site of muscle growth was at the end of the muscle fibre. He was not primarily concerned with tendon growth, but he recorded his impression that the growth of the tendinous part of the myomere also occurred mainly at its junction with the muscle.

A third method of investigation, which forms the basis of this paper, is to mark tendons along their length in young animals and to observe whether the marks become separated during growth. This is an adaptation of Hunter's fundamental experiments on bone growth. A similar method was used by Warwick and Wiles (1934) to study the growth of periosteum. They made marks with indian ink along the periosteum of young rabbits and measured the distance between the marks at the time of the operation and again after an appropriate period of growth. They were able to demonstrate that growth of periosteum occurs along the whole length of the bone and not merely at the region of the epiphysial cartilage. In this investigation the same method has been applied to the study of tendon growth. Small marks were made with indian ink along the length of various tendons in young rabbits. The distance between them was measured at the time of operation and after death two to five months later; and the amount of growth and its situation was determined. 


\section{EXPERIMENTAL MATERIAL AND METHODS}

Rabbits about one month old were used. These are readily obtainable, their tendons are of a convenient size, and although growing rapidly they stand the operation well. The tendons most convenient for study, on account of accessibility and adequate length, were those of tibialis anterior, extensor hallucis longus and the peroneal muscles. Muscles ( $c$.g.. gastrocnemius) whose fibres have a complicated junction pattern with the tendon were avoided, because interpreting growth processes near the muscle-tencon junction would have been more difficult. Since the intention in this first series of experiments was to secure qualitative rather than quantitative data, no special precautions were taken to ensure that all the animals used were of precisely the same breed, age and size. Only a small series of rabbits was used for each particular tendon studied. It was hoped that a fairly general picture of the method of tendon growth would result from using a variety of different tendons.

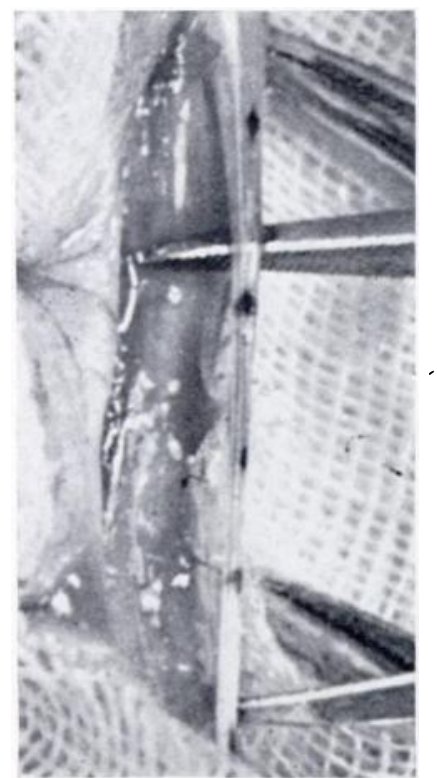

FIG. 1

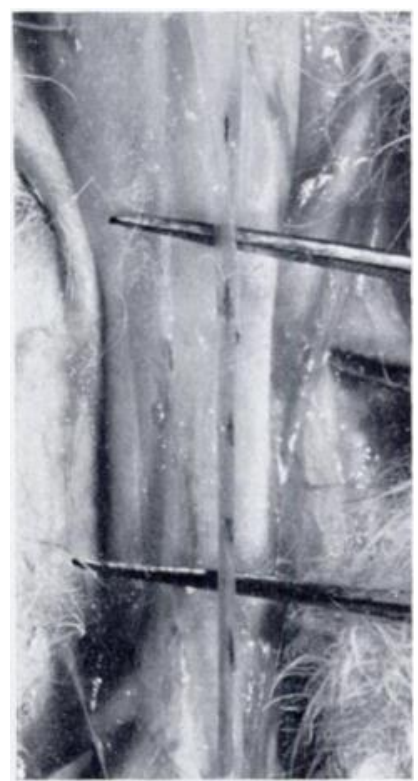

FIG. 2

Figure 1, a photograph taken during operation on a five-week-old rabbit, shows indian ink marks along the tendon of extensor hallucis longus. (Approximately $\times \mathbf{3 \cdot 5}$.) Figure 2 shows the tendon eleven weeks later: the same indian ink marks can be seen. (Approximately $\therefore$.)

Operative technique-Under general anaesthesia the tendon was exposed through a skin incision and the tendon sheath incised. Small marks were made with indian ink at intervals along the length of the tendon by means of a fine needle inserted so that some of the ink was deposited within the tendon substance. Before the tendon was marked its surface was dried with a swab to prevent spreading of the ink in the surface film of fluid. The ink marks were usually about one millimetre in diameter. The distance between the marks was measured with fine dividers on a metal ruler graduated to half a millimetre (Figs. 1 and 2 ). In some animals a pin was inserted into the bone as a fixed marker. Any movement of the tendon relative to the bone during growth was thus determined. Only the skin incision was sutured: natural repair of the tendon sheath occurred rapidly without suture. The rabbits were killed after eight to twenty-two weeks, the tendons examined and the distances between the ink marks measured to determine the growth that had occurred in the segments of tendon between the marks. The ink marks rarely faded; they tended to spread, but only very slightly, and measurements could be taken from the centres. Some control experiments are described overleaf.

voL. 32 B, NO. 2, MAY 1950 
Control experiments. Gross morphology of the experimental tendon-The length of the tendon under investigation was compared after death with its normal counterpart from the opposite limb. In no case was the difference more than two millimetres, though the tendon which had been operated on was sometimes slightly thinner than its fellow. In all cases the arrangement of muscle fibres at the musculo-tendinous junction appeared unaltered; the girth and length of the muscle belly appeared normal. The tendon sheath was found to have regained its normal macroscopic appearance; there were no adhesions limiting the movement of the muscle or tendon. A few cases of wound infection were excluded from the investigation.

Microscopic structure of the tendons-The tendon under investigation and the opposite normal tendon were examined histologically after death. Further, six young animals were killed at intervals of from seven to forty-three days after operation to determine whether there was evidence of abnormal changes in the structure of the tendon in the early post-operative period. Histological examination in these animals showed evidence of an increase in the number of fibroblast nuclei towards the surface of the tendon (Fig. 3) about ten days after operation, but no abnormality of the deeper fibres of the tendon was apparent. Control experiments on rabbits killed two weeks after operation indicated that there had been no appreciable tendon growth in that time. Since the post-operative cellular reaction at the surface of the tendon had usually subsided by the end of the second week, its possible effect on tendon growth can be discounted. Examination of specimens killed ten weeks after operation showed no important differences between the tendon under investigation and the control tendon (Figs. 4 and 5). Histological examination of the marked areas showed remarkably little tissue reaction around the ink (Figs. 6 and 7 ) even when the tendon was examined at a short interval after the marks were made.

Operations on adult rabbits - In order to eliminate the possibility that the operation on the tendons might lead to stretching, experiments were undertaken on the tendon of tibialis anterior in four adult rabbits. Marks were placed on the tendon and the intervals measured. The rabbits were killed after eight weeks and the measurements repeated (Fig. 8). It is clear that the distance between the ink marks has not altered significantly in the eight weeks elapsing between operation and death, and there is thus no evidence that post-operative stretching of the tendons occurs. In this experiment the difference between the first and second measurements was never greater than half a millimetre. This suggests that the method can be relied upon as sufficiently accurate for our purpose.

To summarise-These control experiments suggested that that the operation caused no significant pathological change in a tendon, and there was no positive evidence that the normal growth of the tendon was disturbed. Technique was shown to be adequately accurate.

\section{RESULTS}

The results will be classified according to the tendon studied.

Tibialis anterior-Six rabbits were used. All were about five weeks old at operation. The interval between operation and death varied from eight to fifteen weeks. The tendon was marked at intervals from the muscle-tendon junction to within about ten millimetres of the insertion. The number of marks varied from four to five, and the distance between them from two and a half to nine and a half millimetres. The results are shown in Figure 9. In every case the first mark was made on the muscle tendon-junction and this is represented in the figure by the points joined by the horizontal line.

The amount of tendon growth varied considerably in the different rabbits, but in all cases the distances between the marks increased. Interstitial growth had therefore taken place along the whole length of the tendon marked. It is evident, however, that the rate of growth was not uniform in the different segments. Growth was most rapid near the 


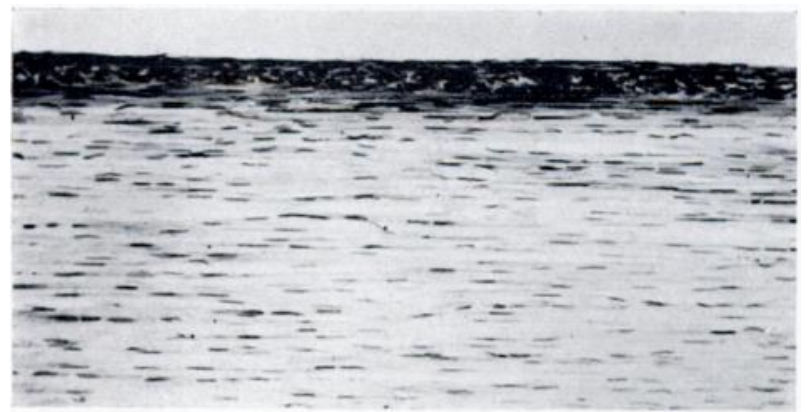

FIG. 3

The surface of a tendon of extensor hallucis longus, which had been exposed for marking at an operation ten days before the rabbit was killed. There is an increase in the number of fibroblast nuclei on the surface. $(\times 130$. $)$

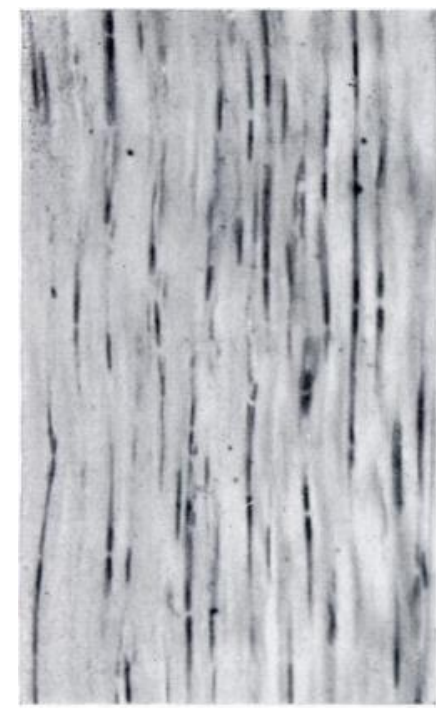

Fig. 4

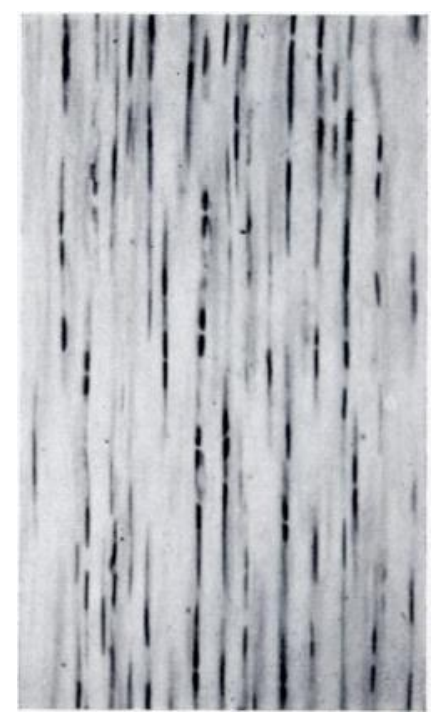

Fig. 5

Figure 4-Tendon of extensor hallucis longus, marked with indian ink at an operation ten weeks before the rabbit was killed, when it was aged seven weeks. The normal appearance of the tissue is evident. $(\times 220$.) Figure 5 - - Tendon of the normal extensor hallucis longus from the opposite leg of the same rabbit. $(\times 220$.

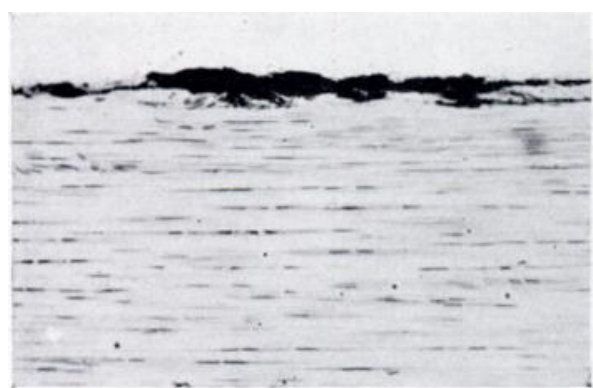

Fig. 6

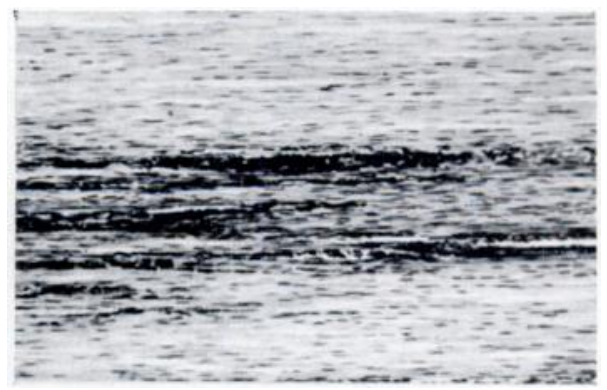

Fig. 7

Figure 6-Indian ink on the surface of a tendon of extensor hallucis longus, marked eleven weeks before the rabbit was killed. $(\times 130$.) Figure 7 -Indian ink in the substance of a tendon of extensor hallucis longus, marked two weeks before the rabbit was killed. The lack of much tissue reaction is evident in either case. $(\times 80$.

vol. $32 \mathrm{~B}$, NO. 2 , MAY 1950 
muscle-tendon junction and fell off more distally along the tendon. This can be seen more clearly in Figure 10. Here the increase in length of each segment (expressed as a percentage of its initial length) is plotted against the distance of the mid-point of the segment from the muscle-tendon junction. The decrease of the growth rate as the measurements are made farther from the muscle-tendon junction is evident; it can be seen also that the most rapid decrease appears to be in the proximal part of the tendon. In the case of rabbit 1 , the segment centred twenty-three millimetres from the muscle-tendon junction shows increased rate of growth. This segment was near the insertion of the tendon and it is possible that the growth rate increases again in this region. Nevertheless the length of the segments was small and an error of half a millimetre could have given rise to a difference of about 10 per cent. in the percentage increase of length. This reading therefore is not regarded as significant.

Extensor hallucis longus (proximal half)-Five rabbits were used. Their ages at the time of operation varied from three and a half to six weeks. The interval between operation and death varied from ten to sixteen weeks. Only the proximal half of the tendon was marked. The first mark was made about three millimetres distal to the muscle-tendon junction. Two to four marks were made, and the distance between them at the time of operation varied from four and a half millimetres to eight and a half millimetres. There was interstitial increase in length in all the segments, but the greatest increase occurred near the muscle-tendon junction (Fig. 11). The percentage increase in length in each segment is shown in Figure 12. The curves in general suggest a more uniform decrease in the rate of growth towards the distal part of the tendon than in the case of the tibialis anterior. However, the segments marked on the tendon of extensor hallucis longus were shorter than those on tibialis anterior, and consequently slight inaccuracies of measurement would be more misleading. The shape of the curves in Figure 12 can therefore be regarded as significant only in showing that the greatest increase in length occurs near the muscle-tendon junction. Extensor hallucis longus (control experiment) - It was considered possible that the markings of a tendon at close intervals might cause a decrease of growth in the affected segment. The fact that the tendon operated on shows a normal total length compared with its counterpart of the opposite limb makes the possibility unlikely. But it does not exclude it entirely since the effect might be compensated by an increase in growth in the unaffected part of the tendon. To test this possibility three further experiments were performed. The tendon of extensor hallucis longus was exposed. Only two or three marks were made, one a few millimetres from the muscle-tendon junction, and the others on the tendon over the distal part of the tibia. The tendon sheath was incised only at these points. Thus a long segment of the tendon was defined and the length of tendon between the marks was undisturbed. The rabbits were five weeks old at the time of operation; they were killed after ten to eleven weeks. The conditions were thus comparable to those of the previous experiments. Figure 13 shows the increase of length in the segment marked. Comparison with Figure 11 indicates that the total growth in several segments of the tendons with multiple marks is closely similar to that which occurred in the single long segment in these experiments. Therefore it appears that the placing of multiple marks along the length of a tendon does not interfere with its growth significantly. Peroneal tendons-Two rabbits were used. One was four weeks and the other eight weeks old at the time of operation. The tendons of peroneus brevis, tertius and quartus were exposed on the dorsum of the foot. All three tendons were marked. Two marks were placed on peroneus brevis to define a segment of tendon the distal end of which was its insertion. The distal marks on peroneus tertius and quartus were placed about eight millimetres from the insertion of the tendons. The rabbits were killed after nineteen to twenty-two weeks. The results are shown in Figure 14. Increase in length occurred in all the segments. Since a fairly short length of tendon was used in only two rabbits it is hardly justifiable to draw quantitative conclusions with regard to the growth rate. But the figures indicate that interstitial growth in the length of a tendon occurs as far distally as its insertion. 

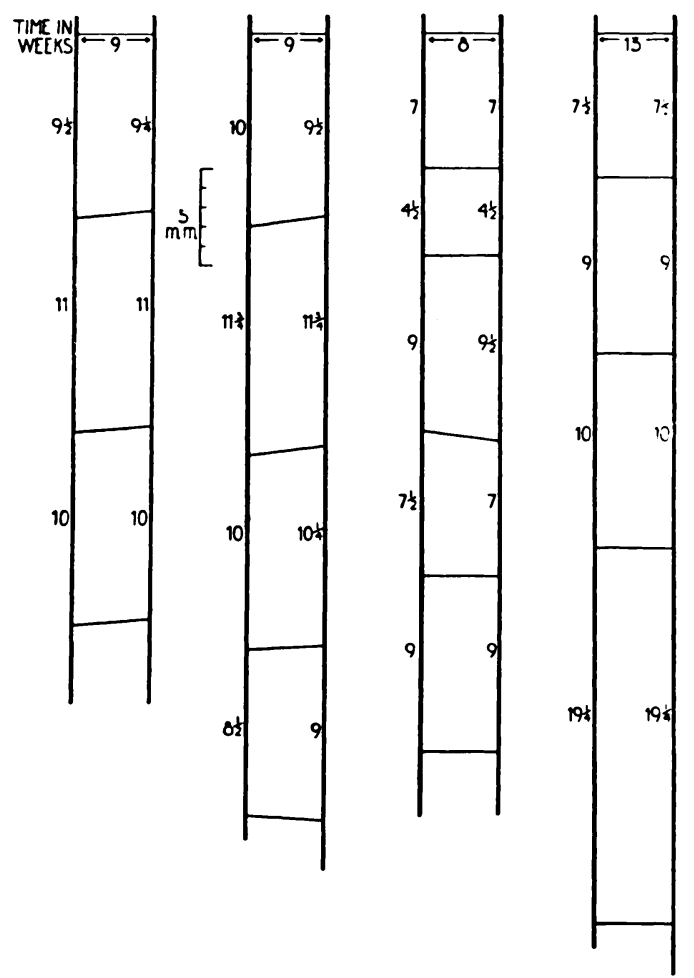

FIG. 8

Diagram illustrating the lack of stretching of the marked tendons of tibialis anterior of four adult rabbits, which were killed about ten weeks after the operation. The vertical lines are in pairs, the first member of each pair representing the tenclon at the time of operation and the second at death. Each of the four pairs represents one of the experimental rabbits. Horizontal or oblique lines join the positions of each ink mark on the tendon at the time of operation and at death. (In Figs. 9.

11 and 13, similar conventions are used.) All the diagrams are drawn to scale and hence show the amount which each segment of the tendon has grown.
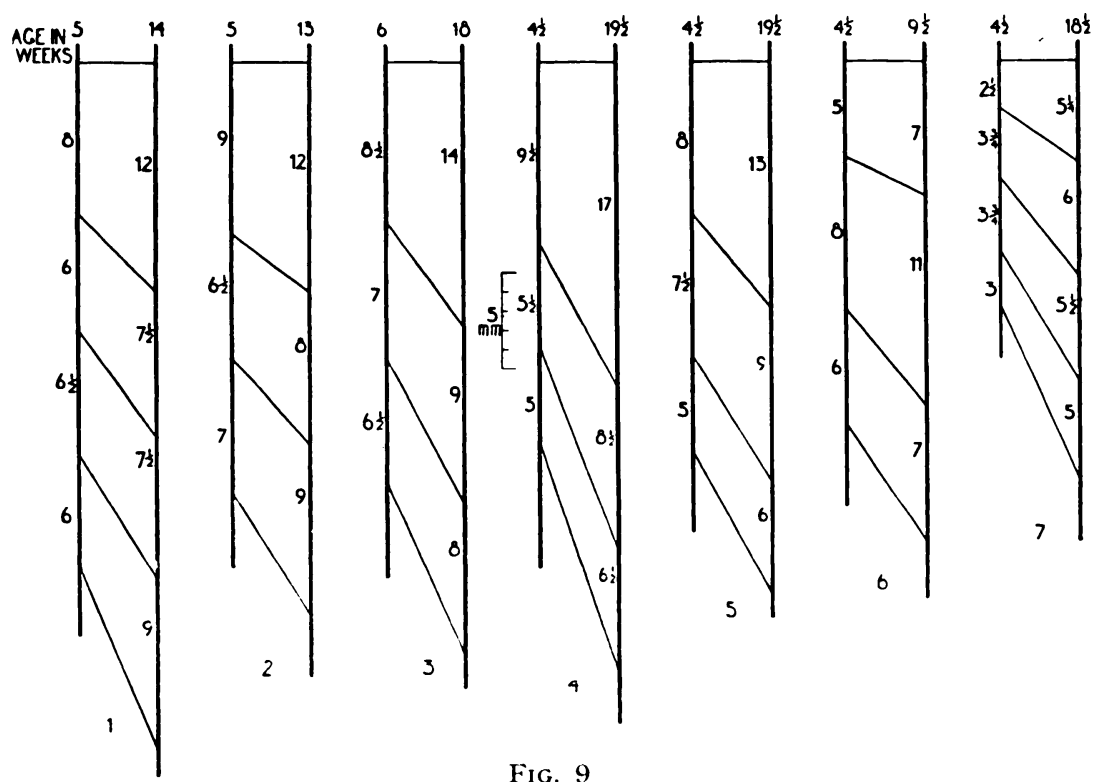

Diagram illustrating the longitudinal growth of the marked tendons of tibialis anterior in seven young rabbits.

VOL. $32 \mathrm{~B}$, NO. 2 , MAY 1950 


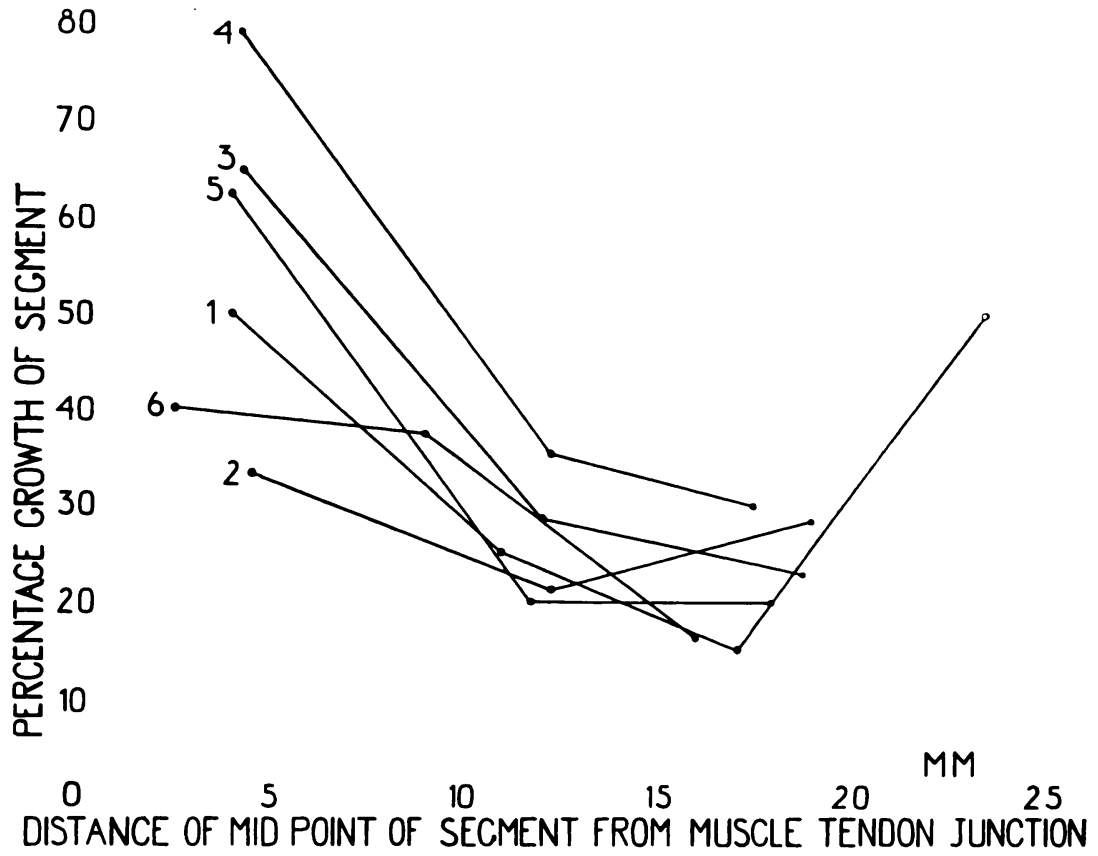

FIG. 10

Diagram illustrating the amount of growth in the various segments marked in tendons 1 to 6 of Figure 9 . The number by the side of each curve refers to the tendon, which can be identified in Figure 9 by the number beneath each pair of vertical lines.

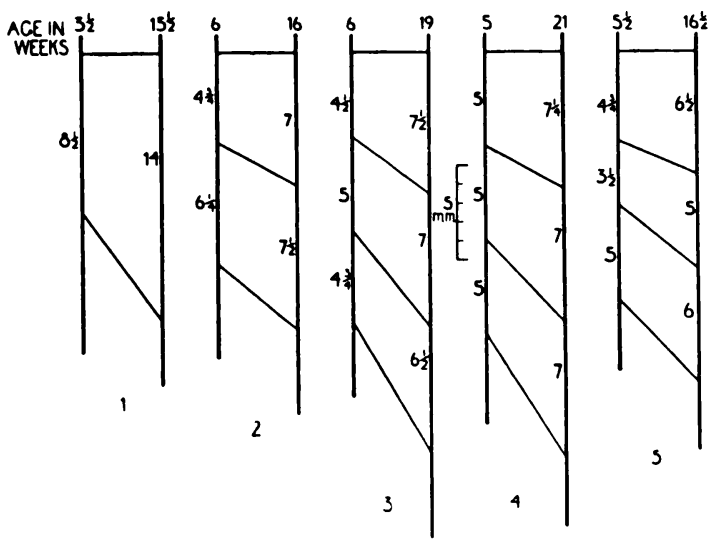

FIG. 11

Diagram illustrating the longitudinal growth of the marked tendons of extensor hallucis longus in five young rabbits. 


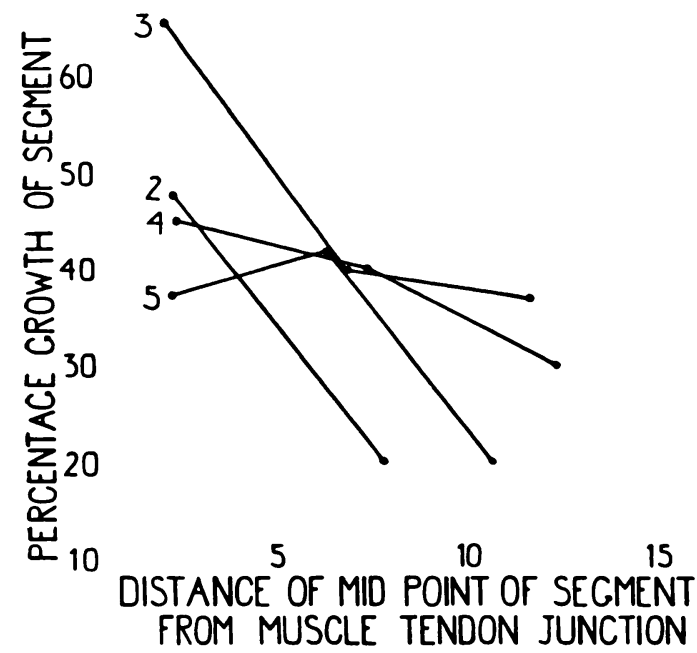

FIG. 12

Diagram illustrating the amount of growth in the various segments marked in tendons 2 to 5 of Figure 11. The number by the side of each curve refers to the tendon, which can be identified in Figure 11 by the number beneath each pair of vertical lines.

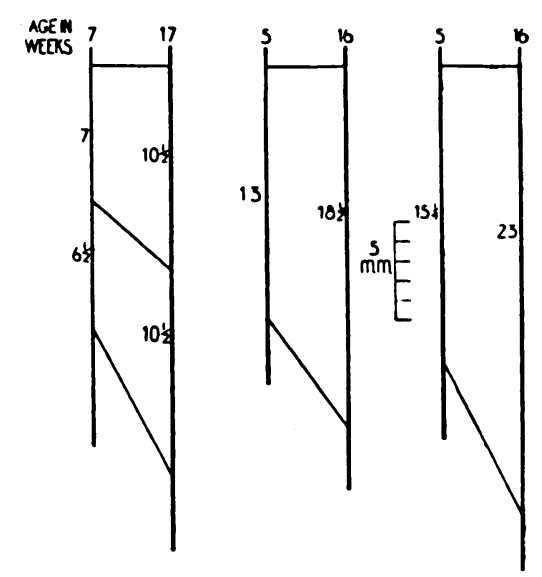

FIG. 13

Diagram illustrating the longitudinal growth of tendons of extensor hallucis longus in three young rabbits. The tendon lying between the ink marks had not been interfered with. For comparison with Figure 11 .
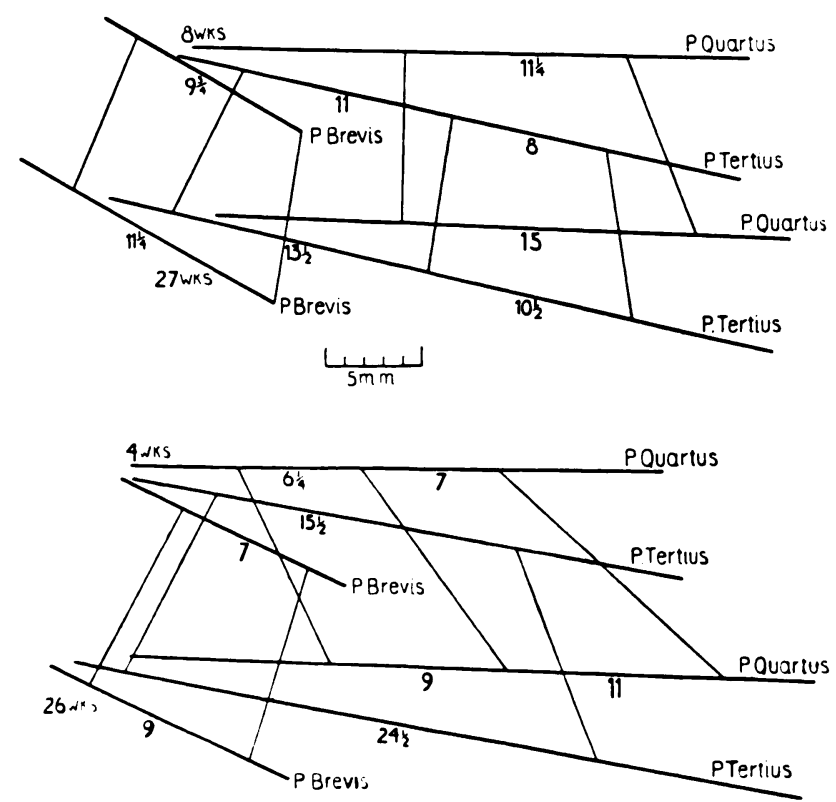

FIG. 14

Diagram illustrating the longitudinal growth of the peroneal tendons lying along the dorsum of the foot in two young rabbits. 


\section{DISCUSSION}

These experiments show that interstitial longitudinal growth occurs throughout the whole length of the tendon in young rabbits. The greatest increase in length occurs near the muscle-tendon junction and there is a gradient of decreasing rate of growth away from this region. It is possible that the rate of growth increases again near the insertion of the tendon; but further experiments are necessary to determine this conclusively. There is no reason to suppose that the growth of tendons follows a different pattern in other mammals, but here also confirmatory experiments are required.

The experiments that have been undertaken do not afford any evidence how a tendon can increase in length interstitially, and it would be unwise to speculate on this point until more detailed knowledge is available concerning the histological structure of tendons and the ultimate structure of collagen. A number of problems remain unsolved. For example, there is doubt whether a muscle fibre is directly continuous with the collagen fibre at the muscle-tendon junction; whether the collagen fibre of the tendon always continues into the bone at its insertion; and whether a single collagen fibre runs throughout the whole length of the tendon. All these points are relevant to the problem of tendon growth. It has yet to be determined whether, as might be expected, a tendon lengthens by accretion of collagenous tissue at the free ends of the tendon fibre (if these occur), or at the junction with the muscle, or at the insertion into the bone. A further possibility is that increase in length is produced by the sliding of tendon fibres over one another-a mechanism that could only operate if, as seems probable, all the fibres do not extend uninterruptedly along the whole length of the tendon.

It is known that the relative and absolute lengths of muscle and tendon in the limbs of the adult animal have a relation to the contractile excursion required of the muscle (Frohse and Fränkel 1908). Alterations in the necessary excursion, such as may be caused by skeletal deformities, result in modifications of the muscles and tendons concerned. Very little is known, however, of the immediate factors determining the muscle-tendon ratio during normal growth or of the factors that determine the modifications made necessary by skeletal deformities occurring in the adult. It is possible that information bearing on these problems might be obtained by marking tendons in both adult and young rabbits and subjecting them to certain experimental modifications. For example, the tension to which a tendon is normally subjected could be altered and the distance between the origin and insertion of the muscle changed. By seeing how the tendon reacted to these new conditions light might be thrown on the factors that determine the normal form of the muscle. Experiments on these lines are now in progress.

\section{SUMMARY}

1. Small indian ink marks were made at intervals along the length of tendons in the limbs of young rabbits, and the distance between the marks was measured during the operation. The rabbits were killed two to three months later, and the amount of longitudinal growth that had occurred was determined by re-measuring the distance between the marks.

2. The experiments showed that the whole of the tendon grows interstitially in length, but that maximal growth occurs near the muscle-tendon junction.

3. Histological examination of the tendons and control experiments involving adult tendons indicated that growth was not significantly interfered with by marking the tendons.

\section{REFERENCES}

Felix, W. (1889): Über Wachsthum der quergestreiften Muskulatur nach Beobachtungen am Menschen. Z. wiss. Zool., 48, 224.

Frohse, F., and Fränkel, M. (1908): Die Muskeln des menschlichen Armes. Handbuch der Anatomie des Menschen (Ed. Bardeleben). Jena: G. Fischer. 
HÄGGQUist, G. (1931): Handbuch der mikroskopischen Anatomie des Menschen (Ed. Möllendorff). Berlin: Julius Springer. Band 2, Teil 3, p. 167.

Haines, R. W. (1932): The laws of muscle and tendon growth. Journal of Anatomy, 66, 578.

Mason, M. L., and Shearon, C. G. (1932): The process of tendon repair. Archives of Surgery, 25, 615.

Prenant, A., Bouin, P., and Maillard, L. (1911): Traité d'Histologie, 2, 326.

SPEIDEL, C. C. (1938): Studies of living muscles. I) Growth, injury and repair of straited muscle, as revealed by prolonged observations of individual fibers in living frog tadpoles. American Journal of Anatomy, 62, 179.

WARWICK, W. T., and Wiles, P. (1934): The growth of the periosteum in long bones. British Journal of Surgery, 22, 169. 